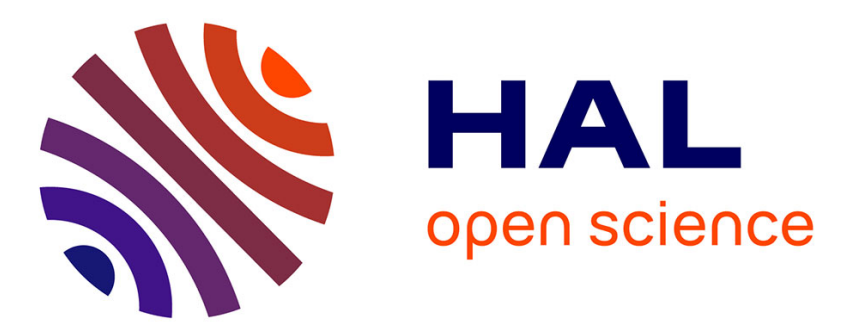

\title{
Critical viscoelastic study of the gelation of silica particles
}

B. Gauthier-Manuel, E. Guyon, S. Roux, S. Gits, F. Lefaucheux

\section{To cite this version:}

B. Gauthier-Manuel, E. Guyon, S. Roux, S. Gits, F. Lefaucheux. Critical viscoelastic study of the gelation of silica particles. Journal de Physique, 1987, 48 (5), pp.869-875. 10.1051/jphys:01987004805086900 . jpa-00210506

\section{HAL Id: jpa-00210506 https://hal.science/jpa-00210506}

Submitted on 1 Jan 1987

HAL is a multi-disciplinary open access archive for the deposit and dissemination of scientific research documents, whether they are published or not. The documents may come from teaching and research institutions in France or abroad, or from public or private research centers.
L'archive ouverte pluridisciplinaire HAL, est destinée au dépôt et à la diffusion de documents scientifiques de niveau recherche, publiés ou non, émanant des établissements d'enseignement et de recherche français ou étrangers, des laboratoires publics ou privés. 
Classification

Physics Abstracts

$82.70-46.60 \mathrm{~B}-82.35$

\title{
Critical viscoelastic study of the gelation of silica particles
}

\author{
B. Gauthier-Manuel, E. Guyon $(*)$, S. Roux $(*)$, S. Gits $\left(^{+}\right)$and F. Lefaucheux $\left({ }^{+}\right)$ \\ Laboratoire d'Electrochimie des Solides UA 0436, Faculté des Sciences et des Techniques, \\ 25030 Besançon, France \\ (*) Laboratoire H.M.P. - UA 857, E.S.P.C.I., 10, rue Vauquelin, 75231 Paris Cedex 05, France \\ $\left({ }^{+}\right)$Laboratoire de Minéralogie-Cristallographie, Universités Paris VI-Paris VII, 4, place Jussieu T16, \\ 75230 Paris Cedex 05, France
}

(Reçu le 7 juillet 1986, révisé le 29 décembre, accepté le 9 janvier 1987)

\begin{abstract}
Résumé. - Nous présentons une analyse de comportement critique de la viscosité et du module élastique lors de la gélification des particules de silice. Deux systèmes sont étudiés : gélification dans une solution de $\mathrm{NaCl}$ ou dans l'eau pure. Les expériences montrent une transition d'un régime d'élasticité entropique au voisinage du seuil de gélation vers un régime d'élasticité «mécanique » au-delà du seuil.

Abstract. - We present an analysis of the critical behaviour of viscosity and elastic modulus during the gelation of silica particles. Two systems are studied : gelation in $\mathrm{NaCl}$ solutions and in pure water. The experiments show a cross-over between entropic elasticity near the gelation threshold and " mechanical» elasticity farther from it.
\end{abstract}

\section{Introduction.}

The study of the sol-gel transition has stimulated a considerable interest from the theoretical as well as experimental side [1] in connection with models of the physics of random matter, and also numerical simulations of the problem [2]. We present in this paper rheological measurement during the gelation process of silica particles. The transition is a critical phenomenon characterized by a threshold where an infinite three-dimensional polymeric cluster first exists across the polymer solution. In the sol phase, below this threshold, only finite polymeric clusters coexist in the solution. Percolation is an appealing theory to describe the behaviour in the vicinity of the gelation threshold. It can be characterized by critical behaviour of the following variables :

The correlation length, which measures the size of the largest cluster or the mesh size of the macrolinks in the gel phase, reads :

$$
\xi(p) \propto\left[\left(p-p_{\mathrm{c}}\right) / p_{\mathrm{c}}\right]^{-\nu} .
$$

The viscosity of the sol, which diverges as the cluster size increases as the threshold is approached from below, is related to $p_{\mathrm{c}}$ as follows :

$$
\eta(p) \propto\left[\left(p_{\mathrm{c}}-p\right) / p_{\mathrm{c}}\right]^{-k} .
$$

The elasticity of the gel, which increases continuously from zero due to the formation of the infinite cluster above threshold, can be described as :

$$
E(p) \propto\left[\left(p-p_{\mathrm{c}}\right) / p_{\mathrm{c}}\right]^{\mu} .
$$

The control parameter $p$ can be taken as proportional to the amount of three-dimensional bonding. In chemical gels where the reaction proceeds regularly with time $T$, the ratio $\left(p-p_{\mathrm{c}}\right) / p_{\mathrm{c}}$ can be replaced by $\left(T-T_{\mathrm{c}}\right) / T_{\mathrm{c}}$, near the critical gelation time $T_{\mathrm{c}}$.

The critical exponents $[\nu, k, \mu]$ are expected to be independent of the local properties of the reaction. In practice, viscoelastic measurements on gels give strongly different values for $k$ and $\mu$ depending on the system studied [1].

At the theoretical level, the mechanical properties of weak elastic media have been the object of many recent works. Two main classes emerge from these studies :

In the first one, the critical behaviour of the elasticity is set in correspondence with that of the conductance of a percolating lattice [3]. The exponent $\mu$ can be identified with that of the electrical percolation problem $t$ ( $\simeq 2.0$ in 3 dimensions). The correspondence assumes an elasticity of a scalar 
nature as for rubber and was shown by Alexander [4] to apply when entropic effects dominate as in the case for long flexible chains.

The second one describes the elastic problem in mechanical terms (e.g. an incomplete lattice of springs with angular force between bonds) [5]. This vector percolation approach leads to a critical exponent $\tau$ larger than $t$. Upper and lower bounds for $\tau$ were derived from geometrical arguments based on the structure of the infinite lattice [5, 7]. They confirm the value for $\tau$ obtained with experiments on macroscopic systems and numerical simulation [8] $(1+\nu d<\tau \simeq(3.5$ to 4$) \leqslant t+2 \nu)$. The application to gels would assume that the dominant contributions are of mechanical origin such as would be obtained if angular elasticity were relevant.

Previous results we had obtained on chemical gels [12] produced by copolymerization of monoand bis-acrylamide were consistent with a scalar elasticity exponent : $\mu \simeq 1.8$ to 2.1 in a critical range $10^{4}<\left(p-p_{\mathrm{c}}\right) / p_{\mathrm{c}}<10^{-2}$. The chains between macrolinks are too flexible to provide any mechanical contribution to the elasticity. On the other hand results by Adam et al. [13] on polycondensation of polyols and diisocyanate lead to an upper value $\mu=3.2 \pm 0.6$ in a critical range $5 \times 10^{-3}<$ $\left(p-p_{\mathrm{c}}\right) / p_{\mathrm{c}}<5 \times 10^{-2}$ in better agreement with mechanical elasticity.

Alexander [4] justified the scalar nature of entropic elasticity in the case of high initial stresses in a macromolecular network. They could be due to osmotic pressure which prevents the collapse of the polymeric lattice. Following these lines [10], a crossover is to be expected from a «scalar" to a «vector" nature of elasticity when the system becomes more and more cross-linked (i.e. as one gets away from the gel-point).

Closely related to these ideas, result of Webman [9] about large deformation in an elastic percolation model would lead to a similar cross-over prediction if the high strain of the chains is attributed to the interaction between the molecular network and the sol phase (e. g. through osmotic pressure).

A first result showing such a cross-over was presented recently by Tokita $e$ al. [11]. They fitted dynamic shear moduli of case in micelle gels with a formula

$$
C\left[p-p_{\mathrm{c}} / p_{\mathrm{c}}\right]^{t}+C^{\prime}\left[\left(p-p_{\mathrm{c}}\right) / p_{\mathrm{c}}\right]^{\tau}
$$

such that the elasticity is scalar near threshold (exponent $t$ ), and vector further from threshold (exponent $\tau$ ) [14].

The present experimental study was carried out on gels obtained by aggregation of silica particles. By controlling the aggregation and growth processes of the grains, one can adjust the local flexibility of the chains. A crossover effect taking place in a narrow range of parameters, similar to that found in [11] is found and analysed in terms of the recent theoretical models.

\section{Preparation.}

Silica particles in aqueous solution are naturally charged and repel each other. The density of surface charges is a function of the $\mathrm{pH}$ of the surrounding solution according to the equilibrium reaction

$$
\mathrm{SiOH} \rightleftharpoons \mathrm{SiO}^{-}+\mathrm{H}^{+} ; \quad K_{\mathrm{A}}=10^{-6.8} .
$$

For example, at $\mathrm{pH} 7$, silica particles are negatively charged with one $\mathrm{SiO}^{-}$group per $10^{3} \AA^{2}$ of the surface [19] the isoelectric point is obtained at $\mathrm{pH} 2$ (value of the $\mathrm{pH}$ which gives a neutral surface to the particle). For $\mathrm{pH}$ lower than 2 , silica is positively charged.

The surface charge density is independent of the size of the particles [19] and causes repulsion between individual particles. When the ionic strength of the solution increased, the surface charges are screened and aggregation of particles is possible. Depending on the original particle size, conditions of aggregation and aging leading to floculation and precipitation or to gelation are obtained. The conditions of $\mathrm{pH}$ and ionic strength needed to obtain a gel are very restrictive and sensitive.

A more suitable way to obtain silica gel without changing any property of the solvent is to synthetise silica particles in situ by polymerization of silicic acid $\mathrm{Si}(\mathrm{OH})_{4}$. Silicic acid is soluble and stable in water when its concentration is lower than 100 ppm (concentration of saturated solution of amorphous silica). For higher concentrations, it spontaneously polymerises. The basic reaction which forms dimers is

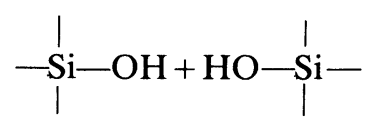<smiles>C[Si](C)(C)O[Si](C)(C)C=O</smiles>

As the reaction proceeds, it leads to the formation of loops and, at the macroscopic level, to compact particles. The property of silica particles to be more soluble when their radius of curvature is lower leads to a relatively monodisperse set of charged spherical particles with a radius of the order of $1 \mathrm{~nm}$.

The further evolution of this system depends on the nature of the surrounding medium. Each particle can either grow in size or aggregate with another particle by Brownian collisions. If salt is present in the solution, the charges are screened and aggregation occurs. This leads to a silica gel made of a three dimensional array of chains of small particles. In the absence of salt in solution, particles grow before they aggregate. Such a gel is made of larger particles in smaller number. Thus the ionic strength of the medium is a very sensitive parameter to control the 
relative rate of aggregation versus growth of silica particles and, consequently, to study the importance of entropic and purely mechanical contributions in the (mechanical) percolation framework.

The reaction scheme used here can be described as follows : Monosilicic acid is synthetized in situ from tetramethoxysilane (T.M.S.) according to the reaction :<smiles>CO[Si](OC)(OC)OC</smiles><smiles>C[14CH2]O[Si](O)(O)O</smiles>

by vigorously stirring (T.M.S.) $0.05 \mathrm{w} / \mathrm{w}$ in solution. The system is set in a thermostated bath and reaction proceeds by itself. A gel is obtained after many hours for a temperature in the range $10-20{ }^{\circ} \mathrm{C}$. Two solvents are used : pure water (milli-Q-grade) and a $0.5 \mathrm{M} \mathrm{NaCl}$ solution in order to study the influence of the ionic strength of the solution on the gel obtained.

\section{Mechanical study.}

We performed measurement of the evolution of the mechanical behaviour of these systems as a function of the reaction time. The origin of time $T=0$ is taken when the system is put in the thermostated bath. The observed behaviour is very similar to previous experiments on the chemical gelation of the acrylamide bis acrylamide system [15].

The figures $1 \mathrm{~A}$ and $\mathrm{B}$ give the evolution of the viscosity and elasticity as a function of the time $T$ for the two solvents used.

After a long period of time showing no appreciable variation of the viscosity, we see a sharp increase in the viscosity followed by the observation of an elastic modulus which increases continuously from zero with time. This change in mechanical behaviour, characteristic of the sol-gel transition, takes place at a critical time $T_{\text {c }}$.

The experiments are done with a «sphererheometer " developed by one of us (BGM) [16] which allows accurate measurements with very low shear rates $\left(10^{-4} \mathrm{~s}^{-1}\right)$ for a large range of viscosity and elasticity. We can follow continuously the evolution of the mechanical state of the system when it goes from a viscous liquid state to an elastic solid one. A magnetic ( $1 \mathrm{~mm}$ diameter) sphere is held within the medium in levitation by an external servocontrolled magnetic field. In static conditions the magnetic force exactly balances the apparent weight of the sphere (corrected by buoancy). The measurements are done as follows. The cell containing the surrounding medium moves vertically at a constant velocity $U$ during a time $T_{\text {exp }}$. An additional force adds or subtracts from the apparent weight (depend-

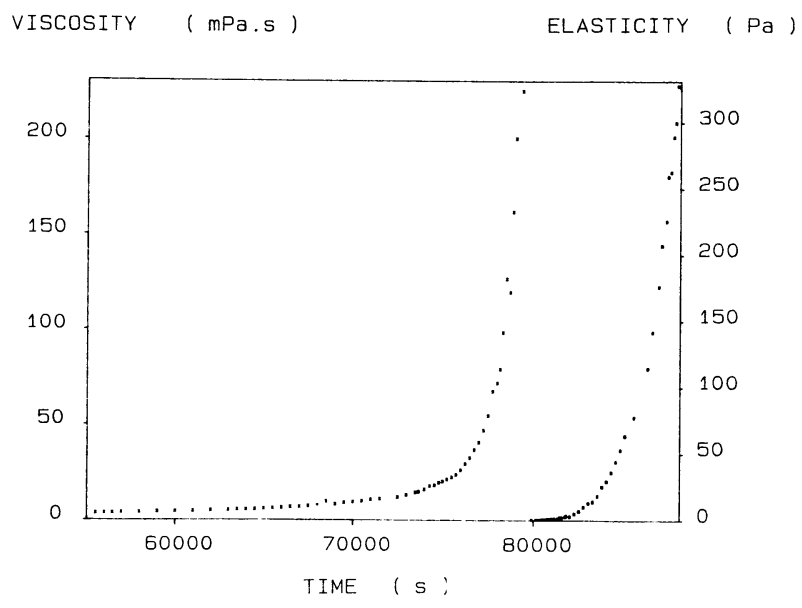

Fig. 1A. - Evolution of viscosity $(\times)$ and elasticity ( $\square)$ vs. time for gel I ( $\mathrm{NaCl}$ solution).

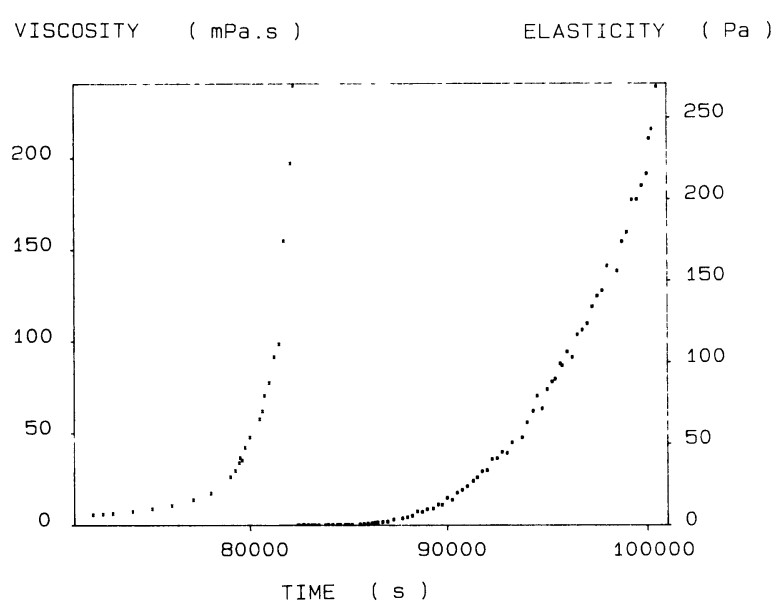

Fig. 1B. - i.e. for gel II (pure water) : viscosity $(x)$; elasticity (ם).

ing of the sign of $U$ ) and the control current varies to keep the sphere fixed. This variation measure the rheological property of the medium.

In the case of a pure viscous fluid the variation of the control current is proportional to the velocity of the displacement and to the viscosity of the fluid (Stokes force).

In the case of a pure elastic solid (as is the case at the end of the experiment) the variation is proportional to the amplitude of the displacement after the time $T_{\exp }$ and to the shear elastic modulus of the medium. The calibration (the same in the two cases) is deduced from the measurement of Newtonian silicon oil of known viscosity.

Near the gelation threshold we observe a viscoelastic behaviour giving relaxation time on the measurement. The experiments are done at very low frequencies $\left(10^{-3} \mathrm{~Hz}\right)$.

The study of the gelation in $\mathrm{NaCl} 0.5 \mathrm{M}$ is done at a temperature of $10 \pm 0.1{ }^{\circ} \mathrm{C}$ which gives a critical 
time of the order of $80000 \mathrm{~s}$. In pure water, gelation for the same concentration in silica takes a much longer time; we have used a temperature of $20^{\circ} \mathrm{C}$ to obtain a gelation time of the same order as that for the first system.

In the case of gelation in a $\mathrm{NaCl}$ solution, figure 2A (and 2B) display the best fits of $\eta \cdot\left(T_{\mathrm{c}}-T / T_{\mathrm{c}}\right)^{k} \quad$ (and $\left.E \cdot\left(T-T_{\mathrm{c}} / T_{\mathrm{c}}\right)^{-\mu}\right)$ versus $\left(T_{\mathrm{c}}-T / T_{\mathrm{c}}\right)$ (and $\left.\left(T-T_{\mathrm{c}} / T_{\mathrm{c}}\right)\right)$, with $k, \mu$, and $T_{\mathrm{c}}$ being considered as free parameters. The simple scaling laws [2, 3] would predict a constant value in these plots. These kinds of fits are generally used to determine eventual correction to scaling. No correction is needed within the accuracy of our data.

The values of the constants obtained from the fits are

$$
\begin{aligned}
& T_{\mathrm{c}}=79250 \mathrm{~s} \\
& k=1.0 \pm 0.1 \\
& \mu=3.8 \pm 0.2
\end{aligned}
$$

Figure 3A shows the corresponding log-log plot of $E$ and $\eta$ versus $\left(T-T_{\mathrm{c}} / T_{\mathrm{c}}\right)$.

A similar analysis for the system in pure water has been done. Away from threshold (from both sides $\left.\left(T-T_{\mathrm{c}} / T_{\mathrm{c}}\right)>0.03\right)$ the fits give

$$
\begin{aligned}
& T_{\mathrm{c}}=81500 \mathrm{~s} \\
& k=1.1 \pm 0.3 \\
& \mu=3.6 \pm 0.4
\end{aligned}
$$

$\eta((T c-T) / T c)^{k}$

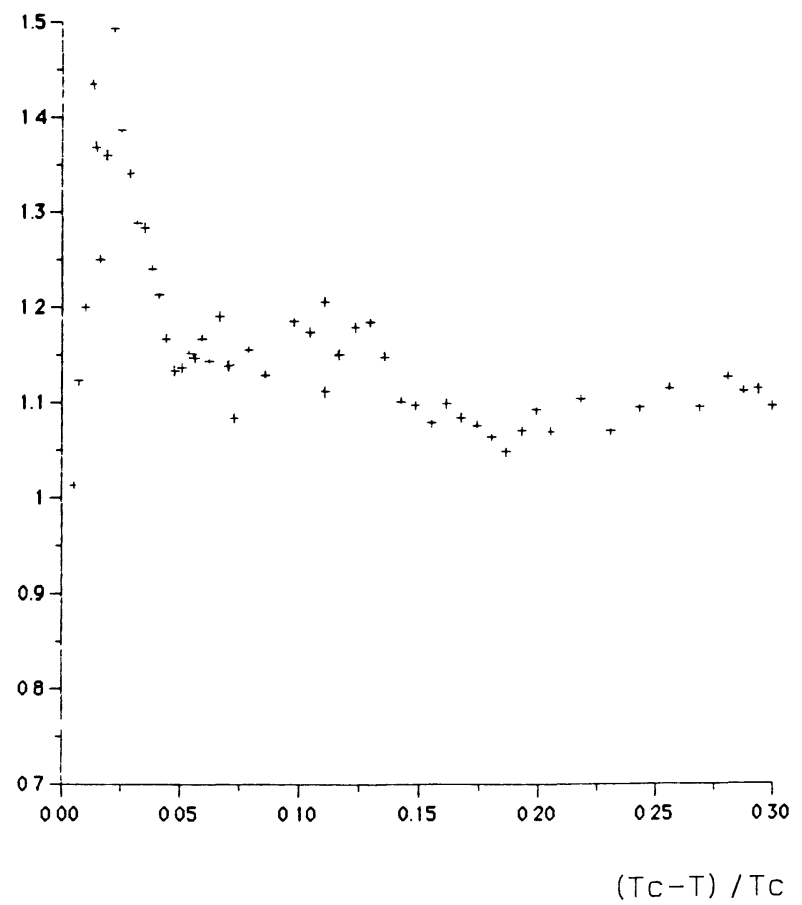

Fig. 2A. - Best fit of $\eta \cdot\left(T-T_{\mathrm{c}} / T_{\mathrm{c}}\right)^{k}$ vs. $T_{\mathrm{c}}-T / T_{\mathrm{c}}$ for gel I for $T_{\mathrm{c}}=79250, k=1.0$.
The determination is less precise than in the former case. Curvature can still be seen on a log-log plot (Fig. 3B). It seems likely that for larger $T$ values we are reaching the limit of the domain of validity of the critical behaviour [3] ; there a cross-over to a non-singular evolution $E$ vs. $T$ should occur smoothly. This might explain the small discrepancies in the data between the two gels.

For $\left(T-T_{\mathrm{c}} / T_{\mathrm{c}}\right)<3 \times 10^{-2}$, a sudden change of behaviour is apparent. Fitting the few points below this limit with any power-law dependence on $T$ would be presumptuous, but for elasticity a slope of about 2 (the value of the scalar exponent in percolation) is consistent with the data presented in figure 3B. A similar, though less marked, change can be seen on the data of figure $3 \mathrm{~A}$.

- Let us note that the cross-over is observed on both sides of the threshold, below it on the viscosity measurement and above it on the elasticity one.

In order to discuss this behaviour let us recall some mechanisms of the aggregation of two silica particles :

If we assume that the interaction between two silica particles is described by the Derjaguin-Landau-Vervey-Overbeck theory [17], the sticking probability of two particles which collide depends on the Debye Hückel screening length $l_{\mathrm{s}}$ (determined by the ionic strength of the solution and by the height of the Coulombic repulsive barrier, which in turn is
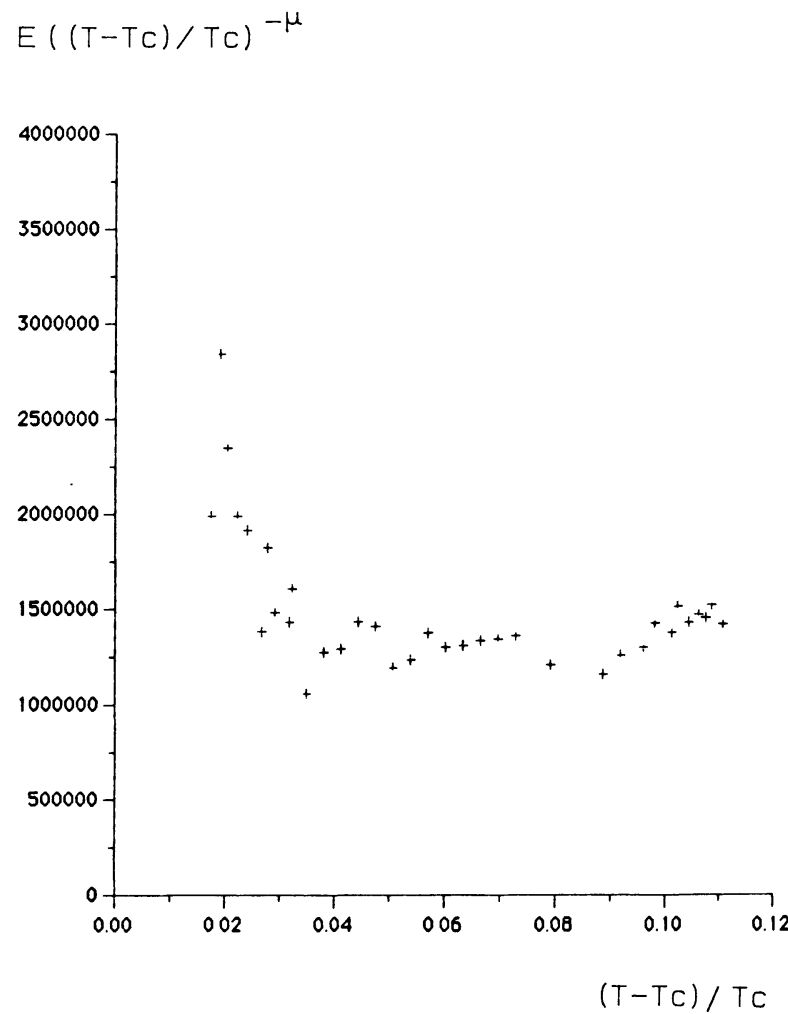

Fig. 2B. - Best fit of $E \cdot\left(T-T_{\mathrm{c}} / T_{\mathrm{c}}\right)^{-\mu} v s . T-T_{\mathrm{c}} / T_{c}$ for gel I for $T_{\mathrm{c}} \mathrm{t}=79250, \mu=3.8$. 


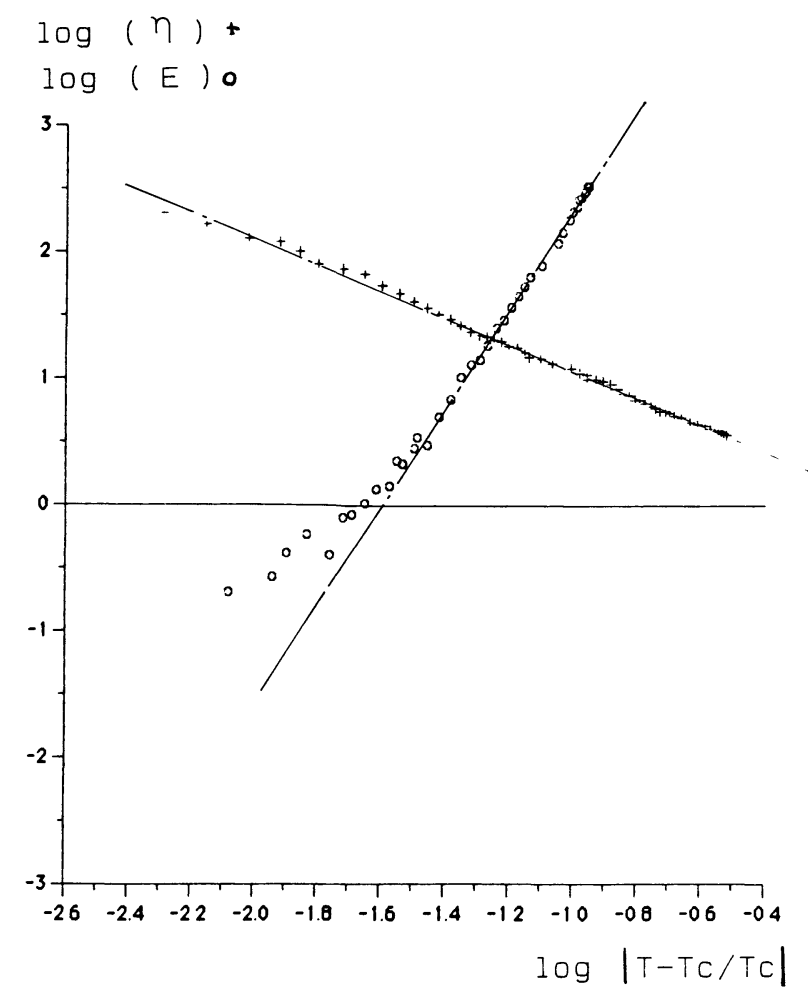

Fig. 3A. - Log-log plot of $E(O)$ and $\eta(+)$ versus $\left(T-T_{\mathrm{c}} / T_{\mathrm{c}}\right)$ for gel I for $T_{\mathrm{c}}=79250$.

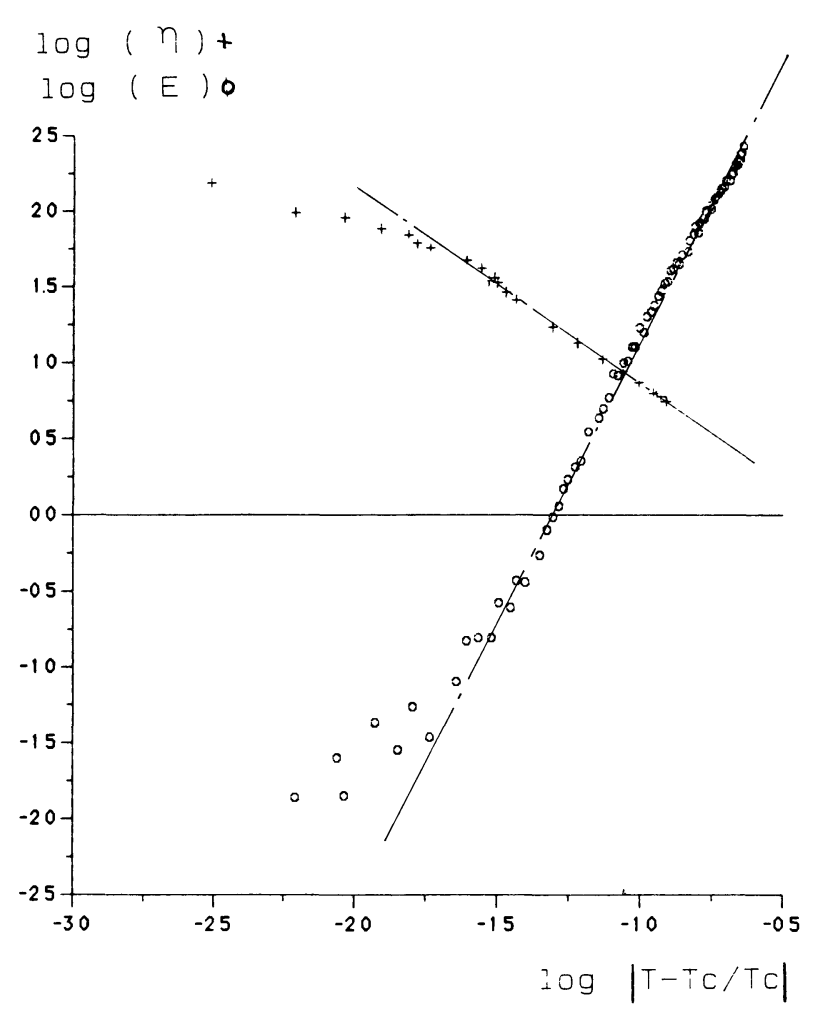

Fig. 3B. - Log-log plot of $E(O)$ and $\eta(+)$ versus $\left(T-T_{\mathrm{c}} / T_{\mathrm{c}}\right)$ for gel II for $T_{\mathrm{c}}=81500$.

fixed by the density of surface charges on the particles). When $l_{\mathrm{s}}$ decreases, aggregation occurs if the short range Van der Waals attraction is greater
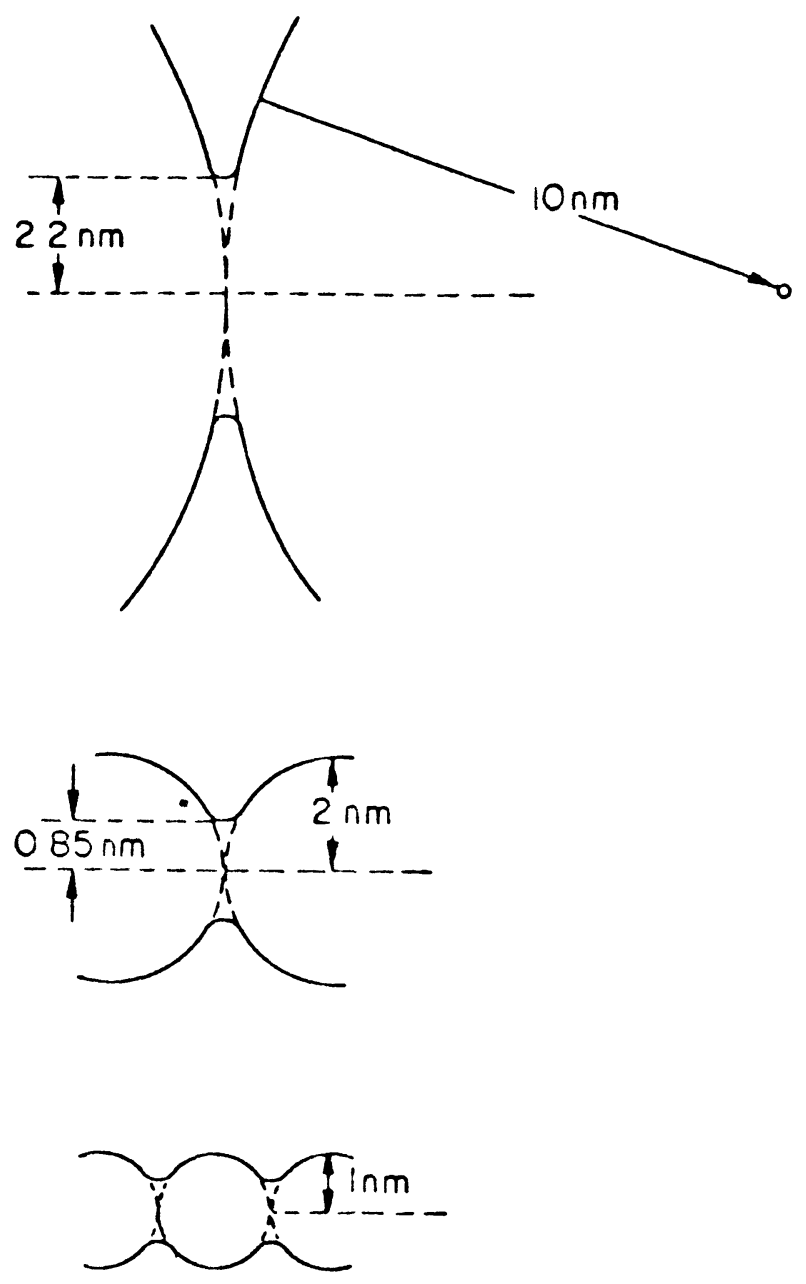

Fig. 4. - Evolution of the size of the neck for different sizes of silica particles (Gels of equal silica concentration). Smaller particles develop greater coalescence and strength, and the structure becomes more fibrillar.

than the Coulombic repulsion. When two particles stick together, instantly the size of the intergranular neck increases because of the initially infinitely small radius of curvature (the rate of increase is governed by the equilibrium law of solubility of silica) until it reaches a limiting value that is a function of the radius of curvature of the initial particle size. This phenomenon, which has been studied in a quantitative way [18] shows that for similarly aged gels, the larger the size of the particles, the smaller the proportion of the neck between the two particles (Fig. 4). This leads to an increased specific rigidity of the chains of small particles by a conversion to a fibrillar structure. The rigidity of chains of small particles corresponding to a fibrillar structure is larger than that of chains of larger particles growing in pure water.

\section{Discussion.}

We propose to explain our results within the framework of percolation theory and suggest that 
the cross-over observed for the gelation corresponds to a change in the nature of the elasticity of the gel. Near threshold, we have rather flexible large particles giving elasticity of entropic nature as in the case of polymer chains of acrylamide, and the elasticity exponent is compatible with a value $\mu \simeq 2$. A scalar exponent is consistent with our analysis [19]. When one gets farther from the gel point, the concentration of crosslinks increases without any (critical) change in the local elastic properties governed by the size of the necks between particles. To explain the crossover we use the picture of «blobs », initially developed for semi-dilute polymer solutions and applied to this problem by Webman [9]. We assume that the osmotic pressure acts as an external stress on the system. At lengths smaller than the blob size $l$, the bondbending forces are large and resist the osmotic pressure ; above size $l$, the bond-bending forces are too small to oppose the osmotic pressure terms and we can see the network as made of entropic chains made with blobs of size $l$. When going from gelation in pure water to that in salt solution, the blob size becomes larger due to the increase of the local rigidity of the chains. Let us note however that this size unit should be fairly independent of the distance to threshold. As one approaches threshold, the correlation length $\xi$ (Eq. (1)), which gives the mesh size of the macrobonds of the gel, tends to increase. In the case of a very large blob size we assume that $\xi$ becomes larger than $l$ very near threshold; the elasticity is that of rigid objects leading to the value $\tau$ of the exponent. On the other hand, for the smaller blob sizes the correlation length would be smaller than $l$ farther from $p_{c}$ where the system would have rigid behaviour. An entropic elasticity and an exponent $t$ would be found near $p_{\mathrm{c}}$. The equality $\xi(p) \simeq 1$ leads to the cross-over value $p^{*}$. This argument suggests that the cross-over should occur farther from $p_{\mathrm{c}}$ in the experiments with pure water than in a salt solution. There is some indication of this result in particular in the behaviour below threshold in the comparison of figures $3 \mathrm{~A}$ and $3 \mathrm{~B}$, but the accurate determination of the cross-over depends crucially on the adjusted fit of the threshold as well as the exponent. In an original analysis of the same data a linear plot giving $E^{1 / \mu}$ as well as $\eta^{1 / k}$ as a function of time $T$ was used and displayed quite clearly the cross-over on the sol as well as gel side for the pure water case (Figs. 33 and 34 of Ref. [20]) whereas the cross-over was practically unobservable on the salt solution results (when using $k=1.1, \mu=3.5$ ). However the pairs of straight lines do not lead to a unique value of $T_{\mathrm{c}}$ unlike the present case and the result is only an indication of the above trend.

Another way to approach this cross-over was proposed by Witten and Kantor [21] based on the study of the limiting radius of stability of aggregates due to thermal vibrations. In this model again, the limiting radius is found to increase with the local rigidity. A rough estimate based on their results gives a value of the order of $10^{4} \AA$ for $l$. Using the order of magnitude equality gives a cross-over distance to threshold $\left(T-T_{\mathrm{c}}\right) / T_{\mathrm{c}} \simeq 10^{-3}$, in reasonable agreement with the experimental finding

$$
\left(T-T_{\mathrm{c}}\right) / T_{\mathrm{c}} \simeq 3 \times 10^{-3} .
$$

The behaviour of the viscosity below threshold shows a similar cross-over effect in the case of pure water solution. At present time, we do not have any reliable interpretation for the two values (inside and outside the cross-over regime) of the exponent $k$ for the viscosity. But if we refer to the geometric analysis of aggregates of silica particles [22] or gold colloids [23] in dilute regime $(<1 \% \mathrm{w} / \mathrm{w})$, a sharp influence of the conditions of aggregation on the fractal dimension is found as one gets from the DLA regime (with a sticking probability $=1$ ) to the chemical regime when screening takes place (with a sticking probability $\ll 1$ ). In the present problem, we would expect that the dominance of entropic or mechanical elasticity would affect strongly the geometry of the finite clusters and most likely the viscous coupling between clusters and with the solvent.

\section{Conclusion.}

The present experiment shows a cross-over regime for the aggregation of silica particles as a function of the distance to threshold. Above the gel point, the cross-over is related to the nature of the elasticity (entropic or mechanical). In particular the crossover between the two regimes of elasticity as well as the values of the exponents consistent with the expected values $t$ and $\tau$ is a strong support of the models. It would be of large interest to study in more detail the range of cross-over on a large range of silica gels prepared with different salinities to confirm the existence of a larger range of scalar elasticity for softer chains. It is also of importance to analyse the corresponding behaviour of the viscosity of the sol phase below threshold. Much less is known on this problem. Quite generally the dynamical properties of colloidal clusters and aggregate is a problem of present interest which should follow the recent developments dealing with their «growth and forms » [24].

\section{Acknowledgments}

We acknowledge fruitful discussions with S. Alexander, H. Hermann, I. Webman. We are indebted to C. D. Mitescu and D. Stauffer for useful advices concerning optimal fits to analyse the data presented in figure 3 . 


\section{References}

[1] Stauffer, D., Coniglio, A., Adam, M., Adv. Polym. Sci. 44 (1982) 105.

[2] Bansil, R., Hermann, H. J., Stauffer, D., Macromolecules 17 (1984) 998.

[3] De Gennes, P. G., J. Physique 40 (1980) 1197.

[4] Alexander, S., J. Physique 45 (1984) 1939.

[5] Kantor, Y., Webman, I., Phys. Rev. Lett. 52 (1984) 1891.

[6] Roux, S., C.R. Heb. Séan. Acad. Sci. 301 Série II (1985) 367 ;

and J. Phys. 19 (1986) L351.

[7] KANTOR, Y., in Scaling phenomena in disordered systems NATO series B 133, R. Pynn \& A. Skejeltorp edit (1986), p. 391.

[8] Zabolitsky, G. J., Bergman, D. J., Stauffer, D., J. Stat. Phys.

[9] Webman, I., Springer Proc. Phys. 5 (1985) 180.

[10] Alexander, S., Springer Proc. Phys. 5 (1985) 162.

[11] Tokita, M., Niki, R., Hikichi, K., J. Chem. Phys. 83 (1985) 256.

[12] Gauthier-Manuel, B., Guyon E., J. Physique Lett. 41 (1980) L-1503.

[13] Adam, M., Delsanti, M., DuRAND, D., Macromolecules 18 (1985) 2285.

[14] As we are describing a cross-over phenomenon we might also use homogeneous forms classically encountered as double power law behaviour see e.g.
Clerc, J. P., Giraud, G., Alexander, S., GuYon, E., Phys. Rev. B 22 (1980) 2489. However this form is much less convenient in the present stage of the numerical analysis, and leads to less accurate results.

[15] Gauthier-Manuel, B., Springer Proc. Phys. 5 (1985) 140.

[16] Gauthier-Manuel, B., Meyer, R., Pierański, P., J. Phys. E (Sci Inst) 17 (1984) 1177.

[17] Verwey, E. J. W., OverbeEK, J. Th. G., Theory of the stability of Lyophobic Colloids (Elsevier New-York) 1948.

[18] ILER, R. K., The chemistry of Silica (Wiley Interscience New-York) 1979.

[19] Mitescu, C. D., Muslof, M. J., J. Physique Lett. 44 (1983) L-679.

[20] Gauthier-Manuel, B., Thèse Paris 1986.

[21] Kantor, Y., Witten, T. A., J. Physique Lett. 45 (1984) L-675.

[22] Schaffer, D. W., Martin, J. E., Wiltzius, P., Cannell, D. S., Phys. Rev. Lett. 52 (1984) 2371.

[23] Weitz, D. A., Huang, J. S., Lin, M. Y., Sung, A. J., Phys. Rev. Lett. 54 (1985) 1416.

[24] Stanley, H. E., Ostrowski, N., ed. On growth and forms (M. Nijhoff pub. Netherland) 1986. 